\title{
Prediction of Relapse or Survival after Resection in Human Hepatomas by DNA Flow Cytometry
}

\author{
Jen-Hwey Chiu, ${ }^{\star \star}$ Hwa-Li Kao, ${ }^{\star}$ Li-Hwa Wu, ${ }^{\star}$ Hwey-May Chang, ${ }^{\star}$ and Wing-Yu Lui ${ }^{\ddagger}$ \\ ${ }^{*}$ Graduate Institute of Clinical Medicine, National Yang-Ming Medical College, Shih-Pai, Taipei, Taiwan, Republic of China; and \\ ${ }^{\ddagger}$ Division of General Surgery, Department of Surgery, Veterans General Hospital, Shih-Pai, Taipei, Taiwan, Republic of China
}

\begin{abstract}
To investigate the change of DNA content and the effect of synthetic phase (S-phase) fraction on hepatocytes and hepatomas, DNA content and S-phase fraction were measured by flow cytometry in human livers and hepatoma tissues. The ploidy status of nontumor parts of resected hepatoma, fetal liver, and focal nodular hyperplasia were diploid, similar to that of the normal liver. Three patterns of DNA ploidy in human hepatoma cells were newly classified, namely, pattern I, diploid tumors; pattern II, aneuploid tumors with single G0/G1 peak; and pattern III, aneuploid tumors with more than one G0/G1 peaks. Among the 130 resectable hepatomas measured for DNA ploidy status, $84(64.6 \%)$ were pattern I, 20 (15.4\%) pattern II, and $26(20 \%)$ pattern III. Multivariate analyses for those 130 patients who underwent hepatic resection showed that, in addition to tumor size, DNA ploidy was another prognostic factor in predicting overall survival and disease-free survival. Patients with small tumors $(<5 \mathrm{~cm})$ had a significantly higher overall survival rate than those with large tumor $(>5$ cm). Patients with pattern III hepatomas had a significantly lower overall survival rate and a higher recurrent rate than did those with pattern I or pattern II tumors. The S-phase fraction was a significant predictor of overall survival rate in patients with pattern II, but not with pattern I, tumors. We conclude that DNA flow-cytometric measurements of ploidy and Sphase fraction are potential important prognostic predictors in patients with resectable hepatomas. (J. Clin. Invest. 1992. 89:539-545.) Key words: DNA ploidy • DNA content $\bullet$ hepatocellular carcinoma - synthetic phase fraction - focal nodular hyperplasia
\end{abstract}

\section{Introduction}

Hepatocellular carcinoma (hepatoma) is a common deadly disease in Asia, especially in Chinese populations. In the Taiwan area, it ranks first in males and third in females as a cause of cancer death (1). Although some hepatomas with a slow growing property have been reported $(2,3)$, the prognosis of hepatomas is very poor. It has been reported that determination of

Address correspondence and reprint requests to Jen-Hwey Chiu, M.D., Ph.D., Division of General Surgery, Department of Surgery, Veterans General Hospital, Shih-Pai, Taipei, Taiwan, Republic of China.

Received for publication 24 June 1991 and in revised form 11 October 1991.

J. Clin. Invest.

(c) The American Society for Clinical Investigation, Inc.

$0021-9738 / 92 / 02 / 0539 / 07 \$ 2.00$

Volume 89, February 1992, 539-545
DNA content is helpful in understanding the biological functions and in predicting the prognosis of neoplasm (4-8). Previously, using cell cycle analysis, studies on rodent liver carcinogenesis showed a substantial increase in the fraction of diploid hepatocytes, both in preneoplastic liver $(9,10)$ and tumors $(11-$ 13). However, ploidy changes in human hepatomas, which have been grouped into diploid and aneuploid status, have been reported to have no correlation with survival of patients with hepatomas who underwent hepatic resection $(14,15)$.

Recently, proliferative capacity estimated by fraction of the synthesis phase of the cell cycle (S-phase ${ }^{1}$ fraction) in DNA flow analysis has been reported to be useful in predicting the prognosis in node-negative breast cancer (16). In addition, using cell cycle analysis and human hepatoma cell lines, we have found that proliferative capacity correlates well with cells of different differentiation stages (Chiu, J. H., et al., manuscript in preparation). It is, therefore, our interest to measure the ploidy change, estimate the proliferative capacity, and evaluate the clinical importance of these measurements in combination with other documented prognostic factors in order to identify patients with resectable hepatomas who may be at high or low risk for tumor recurrence.

In this study, we retrospectively analyzed the DNA ploidy status and proliferative capacity of human liver of benign lesions and 130 resectable hepatomas and used newly classified DNA ploidy pattern to predict biological behavior and survival in patients with resectable hepatomas.

\section{Methods}

\section{Patients and specimens}

From 1985 to 1990 , records of human hepatoma patients in Veterans General Hospital, Taipei, Taiwan, Republic of China, were retrospectively reviewed. 130 patients with surgically resectable hepatomas were eligible for inclusion in this study. Palliative resection, defined as resection with residual tumor proved by any modalities (operative findings, intraoperative ultrasonography or pathology, etc.), were excluded. Information about age of the patient at operation, tumor size, tumor cut margin (the shortest distance between tumor margin and the margin of resected specimen), degree of liver cirrhosis and infection of hepatitis $B$ virus (HBV) were obtained from well documented records. After operation, the patients were followed up at intervals of 2-4 mo with alphafetoprotein, abdominal ultrasonography, computerized tomography scanning, or angiography as indicated. The hepatoma was considered to be recurrent if any two of these four modalities were interpreted as positive findings.

The tumor and nontumor parts of hepatoma specimens and four focal nodular hyperplasia were obtained from resected specimens. 20-

1. Abbreviations used in this paper: $\mathrm{G} 0 / \mathrm{G} 1$, presynthetic growth phase; HBsAg, hepatitis B surface antigen; S-phase, synthetic phase. 
wk and 28-wk fetus livers were obtained according to the regulations of Veterans General Hospital from aborted fetuses, and normal livers from organ donors who died due to car accidents. The fresh samples were kept in liquid nitrogen or $\mathrm{a}-70^{\circ} \mathrm{C}$ freezer.

\section{DNA flow cytometry}

DNA content was analyzed with fresh, frozen tissue or paraffin-embedded samples with some modification as previously described $(17,18)$. In brief, single cell suspensions were prepared from fresh, unfixed surgical specimens or frozen tissue stored at $-70^{\circ} \mathrm{C}$. They were minced into minute pieces, teased, and filtered. The filtrates were allowed to stand on ice for $5 \mathrm{~min}$. Then, the supernatant was filtered, washed with PBS twice, and resuspended in PBS to a concentration of $2-5 \times 10^{6} \mathrm{cells} / \mathrm{ml}$. To the sample was added $0.5 \mathrm{ml}$ ( $\mathrm{vol} / \mathrm{vol}$ ) staining solution A $(50 \mu \mathrm{g} / \mathrm{ml}$ propidium iodide, no. p-4170; Sigma Chemical Co., St. Louis, MO), 3\% polyethyleneglycol 6000 (Art. 807491; Merck, Darmstadt, Germany), 0.1\% Triton X-100, 180 U/ml RNase (No. R-5503; Sigma), 4 $\mathrm{mM}$ citrate buffer $\mathrm{pH} 7.2$, incubated at $37^{\circ} \mathrm{C}$ for $20 \mathrm{~min}$, followed by the addition of solution B ( $\mathrm{vol} / \mathrm{vol})(50 \mu \mathrm{g} / \mathrm{ml}$ propidium iodide, $3 \%$ polyethyleneglycol $6000,0.1 \%$ Triton X-100, $0.4 \mathrm{M} \mathrm{NaCl}, \mathrm{pH} 7.2$ ). The sample was then stored at $4^{\circ} \mathrm{C}$ for at least $1 \mathrm{~h}$ and filtered before being analyzed using a flow cytometer (Epics Profile; Coulter Electronics, Hialeah, FL) supplemented with an Omnichrome 500 series (150 $\mathrm{mW}$; Ominicon, CA), argon ion laser ( $25 \mathrm{~mW}$ at $488 \mathrm{~nm}$ ). Red fluorescence was detected through a 610-nm long pass absorbance filter (3802055; Coulter Electronics). All data were analyzed with off-line software (Cytologic; Coulter Electronics). To obtain cell suspensions from paraffin-embedded specimens, $50-\mu \mathrm{m}$ sections were cut with a microtome. About 1-3 sections were placed in a glass centrifuge tube, dewaxed with two times $100 \%$ xylene, rehydrated in a series of descending concentrations of ethanol ( $3 \mathrm{ml}, 10 \mathrm{~min}$, room temperature), washed with distilled water twice and, finally, resuspended in $1 \mathrm{ml}$ of a solution containing $0.5 \%$ pepsin (p-6887; Sigma), $0.9 \% \mathrm{NaCl}, \mathrm{pH} 1.5$. The samples were then placed in a waterbath, $37^{\circ} \mathrm{C}$, for $30 \mathrm{~min}$. Approximately $10^{5}$ cells were present in the sample. The cell suspension was centrifuged and the pellet was resuspended in the staining solution, followed by the procedures as mentioned above.

DNA content was evaluated as presynthetic growth phase (G0/G1), S-phase, and postsynthetic and mitotic growth phases $(\mathrm{G} 2+\mathrm{M})$. The ploidy status was defined as diploid $(2 \mathrm{~N})$ when $\mathrm{G} 0 / \mathrm{G} 1$ peak was superimposed after the addition of human peripheral blood lymphocytes to the samples with a range from 1.9 to $2.1 \mathrm{~N}$, while aneuploid was defined if the discrete G0/G1 peak(s) was/were outside the diploid range (1.9-2.1 N). The DNA ploidy status of human hepatomas could be further classified into three patterns: pattern I, hepatomas with diploid DNA distribution; pattern II, hepatomas with aneuploid DNA content and containing only one prominent $\mathrm{G} 0 / \mathrm{G} 1$ peak; and pattern III, hepatomas with aneuploid DNA content and containing $>1 \mathrm{G} 0 / \mathrm{G} 1$ peaks. In this study, the coefficient of variation of the G0/G1 half-peak width of single-peak specimens had to be $<5.0-6.0$ for a specimen to be considered interpretable for ploidy status.

\section{Statistical analysis}

The data were analyzed using a statistical package (BMDP Statistical Software, Inc., Los Angeles, CA). The primary end points in this study were disease-free survival and overall survival. Patients who died without documented disease recurrence were included in analysis of overall survival. Disease-free survival was measured between the date of opera-

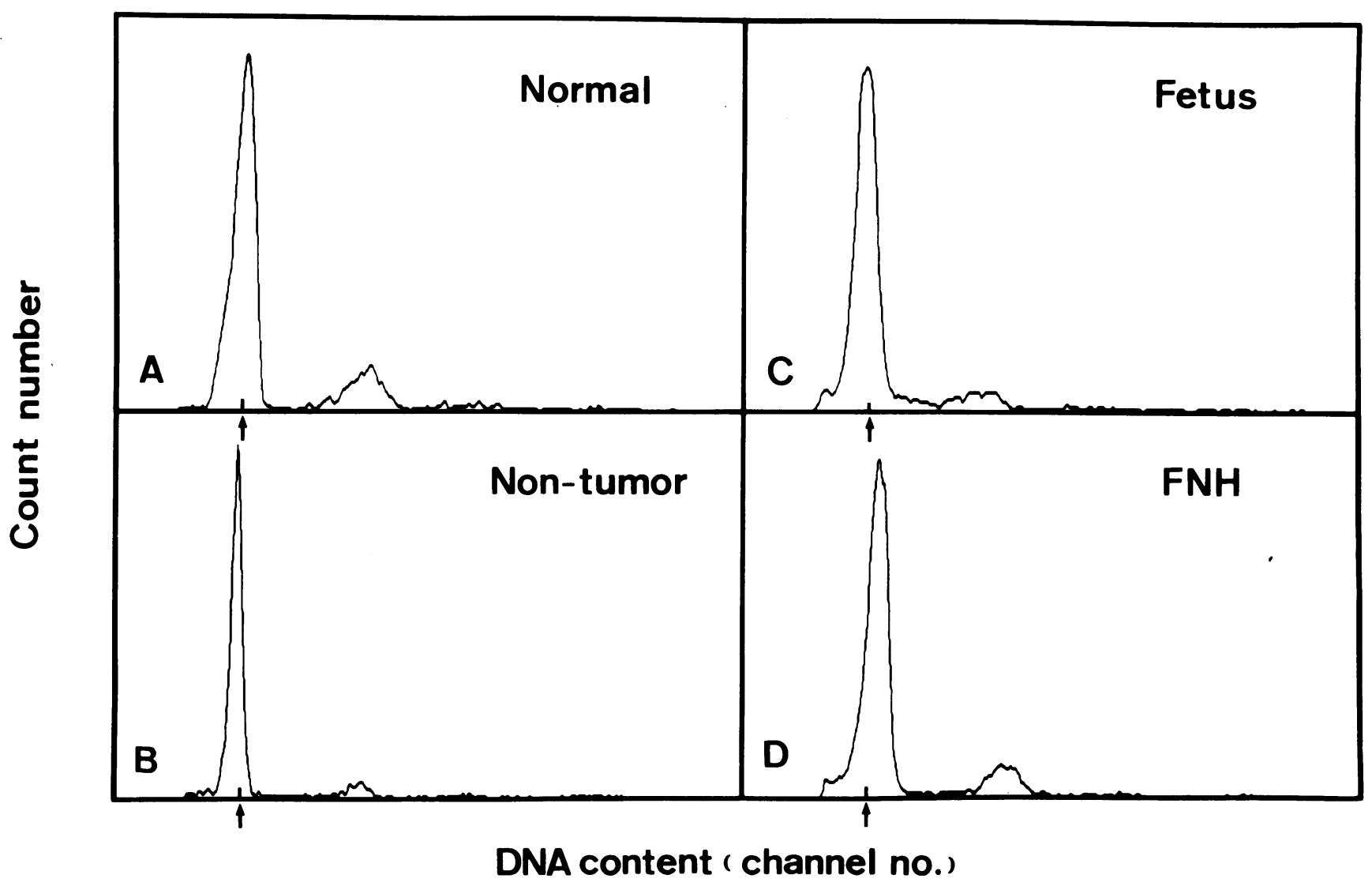

Figure 1. DNA ploidy distribution in nonmalignant liver tissues, including normal liver $(A)$, nontumor parts of resected hepatoma specimens $(B)$, fetal liver $(C)$, and focal nodular hyperplasia $(F N H)(D)$. The specimens were obtained, treated, and the nuclear DNA contents were analyzed with flow cytometry as described in Methods. Arrows indicate relative DNA content corresponding to diploidy (2N). 
tion and date of hepatoma recurrence defined by criteria previously mentioned. Curves for overall survival and disease-free survival were calculated according to the method of Kaplan and Meier (19). Cox's partially nonparametric regression model was used to evaluate the predictive power of various combinations of prognostic factors in a multivariate manner (20). In tumors of pattern I (diploidy) or pattern II (aneuploidy containing single G0/G1 peak), the optimal cutoffs to distinguish DNA content with high S-phase fraction from those with low S-phase fraction were obtained by maximizing the likelihood function with the use of Cox's regression analyses.

\section{Results}

DNA pattern of nonhepatoma livers. Nuclear DNA contents of normal livers, two fetal livers (20 and 28-wk gestation age), four focal nodular hyperplasia, and 78 nontumor parts of hepatoma specimens were analyzed. They were pathologically proved to have no malignant change. The ploidy distribution of nonhepatoma livers including normal (Fig. $1 \mathrm{~A}$ ), nontumor parts of hepatoma specimens resected from hepatoma patients (Fig. 1 $B$ ), fetal livers (Fig. $1 C$ ), and focal nodular hyperplasia (Fig. 1 $D$ ) were all diploid. The percentages of cells in S-phase were similar in these specimens. In nontumor parts, the presence and the degree of liver cirrhosis made no difference in DNA ploidy and DNA content (data not shown).

DNA pattern of hepatoma tissues. 130 specimens with pathologically proved hepatomas were analyzed with flow cytometry and the nuclear DNA ploidy status of these hepatomas was classified into three patterns as described in Methods. Of these 130 specimens, $84(64.6 \%)$ were pattern I (Fig. $2 A$ ), defined as diploidy (1.9-2.1 N); 20 (15.4\%) were pattern II, defined as aneuploidy containing only one G0/G1 peak, either hypoploidy $(<1.9 \mathrm{~N})$ (Fig. $2 \mathrm{~B}$ ) or hyperploidy $(>2.1 \mathrm{~N})$ (Fig. 2 $C)$; and $26(20 \%)$ were pattern III, defined as aneuploidy containing more than one G0/G1 peaks, either diploidy predominant (Fig. $2 D$ ), aneuploidy predominant (Fig. $2 E$ ), or having more than one aneuploid peaks (Fig. $2 F$ ). Only in pattern I and pattern II specimens could the DNA content be further analyzed with S-phase fraction, since in pattern III tumors the S-phase fractions in the multiple G0/G1 peaks were hardly interpretable. Among these 104 hepatomas, the overall mean percentage of cells in S-phase was $5.70 \%$ and a cutoff value $6 \%$ was used to distinguish high from low S-phase hepatomas. In

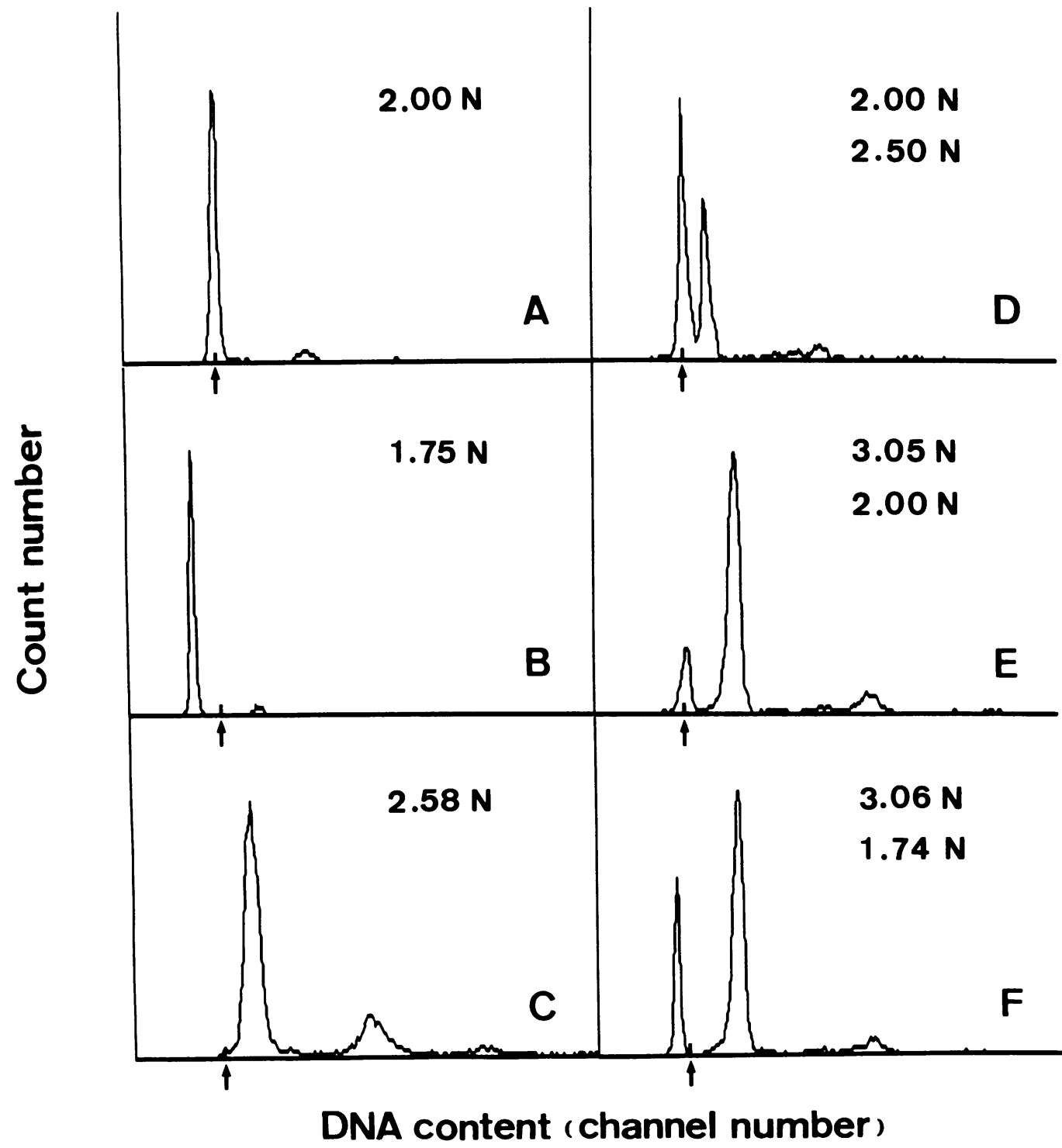

Figure 2. DNA ploidy distribution in human hepatomas. The specimens were treated and the nuclear DNA contents were analyzed with flow cytometry as described in Methods. Three patterns of nuclear DNA ploidy status were newly classified. Pattern I, diploidy (1.9$2.1 \mathrm{~N})(A)$. Pattern II, aneuploidy with one G0/G1 peak, either hypoploidy $(<1.9 \mathrm{~N})(B)$ or hyperploidy $(>2.1$ N) $(C)$. Pattern III, aneuploidy with more than one $\mathrm{G} 0 / \mathrm{G} 1$ peaks, either diploidy predominant $(D)$, aneuploidy predominant $(E)$, or having more than one aneuploid $\mathrm{G} 0 / \mathrm{G} 1$ peaks $(F)$. Arrows indicate relative DNA content corresponding to diploidy $(2 \mathrm{~N})$. 


\begin{tabular}{|c|c|c|c|c|c|c|c|c|}
\hline & \multicolumn{6}{|c|}{ DNA Pattern* } & & \\
\hline & \multicolumn{2}{|c|}{ I } & \multicolumn{2}{|c|}{ II } & \multicolumn{2}{|c|}{ III } & \multicolumn{2}{|c|}{ Total } \\
\hline & No. & Percent & No. & Percent & No. & Percent & No. & Percent \\
\hline No. of cases & 84 & 64.6 & 20 & 15.4 & 26 & 20.0 & 130 & \\
\hline \multicolumn{9}{|l|}{ Age } \\
\hline$\leq 50$ & 15 & 60.0 & 5 & 20.0 & 5 & 20.0 & 25 & 100 \\
\hline$>50$ & 69 & 65.7 & 15 & 14.3 & 21 & 20.0 & 105 & 100 \\
\hline \multicolumn{9}{|l|}{ Tumor size } \\
\hline$\leq 5 \mathrm{~cm}$ & 38 & 65.5 & 12 & 20.7 & 8 & 13.8 & 58 & 100 \\
\hline$>5 \mathrm{~cm}$ & 46 & 63.9 & 8 & 11.1 & 18 & 25.0 & 72 & 100 \\
\hline \multicolumn{9}{|l|}{ Cirrhosis } \\
\hline+ & 62 & 63.2 & 18 & 18.4 & 18 & 18.4 & 98 & 100 \\
\hline- & 22 & 68.8 & 2 & 6.2 & 8 & 25.0 & 32 & 100 \\
\hline \multicolumn{9}{|l|}{ HBsAg } \\
\hline+ & 52 & 65.0 & 14 & 17.5 & 14 & 17.5 & 80 & 100 \\
\hline- & 32 & 64.0 & 6 & 12.0 & 12 & 24.0 & 50 & 100 \\
\hline
\end{tabular}

+, presence; -, absence. * DNA pattern (I, II, III) was defined as described in Methods.

84 hepatomas with pattern I ploidy, 52 were high S-phase tumors and 32 low S-phase tumors, whereas in pattern II hepatomas, 11 were high S-phase tumors and 9 were low S-phase tumors.

DNA pattern and clinical parameters. Specimens from 130 patients who underwent hepatic resection eligible for this study were analyzed by flow cytometry, and their demographic characteristics that could be evaluated are shown in Table I. Among these 130 specimens, $84(64.6 \%)$ were diploid, $72(55.4 \%)$ were $>5 \mathrm{~cm}$ in diameter, and $98(75.4 \%)$ were liver cirrhosis. 105 $(80.8 \%)$ patients were over 50 years of age and $80(61.5 \%)$ showed the presence of hepatitis B surface antigen $(\mathrm{HBsAg}+)$. The median duration of follow-up in the patients still alive at the time of analysis was 48 months. Age, presence of hepatitis B surface antigen, presence of liver cirrhosis, and tumor size were compared with DNA patterns (Table I). No significant differences were noted between DNA patterns and these parameters.

DNA pattern and prognosis. The patient variables were subjected to univariate and multivariate survival analyses in order to identify combinations of factors that could predict overall survival. The variables included in these analyses were ploidy status (patterns I, II, and III), tumor size ( $\leq 5 \mathrm{~cm}$ or $>5 \mathrm{~cm})$, cut margin ( $\leq 1 \mathrm{~cm}$ or $>1 \mathrm{~cm}$ ), liver cirrhosis (yes or no), hepatitis $\mathrm{B}$ antigen status ( $\mathrm{HBsAg}+$ or $\mathrm{HBsAg}-$ ), and the patient's age ( $\leq 50$ or $>50$ ). In both univariate and multivariate analyses, tumor size and ploidy status were the significant factors in predicting overall survival (Table II). We examined tumor size of various cutoffs and found that $5 \mathrm{~cm}$ was the critical cutoff to yield statistically significant differences $(P<0.05)$ between large (tumor size $>5 \mathrm{~cm}$ ) and small (tumor size $\leq 5 \mathrm{~cm}$ ) hepatomas in overall survival. In addition, DNA ploidy status was another factor in determining the prognosis of patients with resectable hepatomas. In this study, DNA ploidy of hepatoma specimens could be grouped into three patterns (Fig. 2). Multivariate analyses of these 130 patients showed that there were statistically significant differences in the overall survival rates between patients with pattern I and pattern III tumors ( $P$ $<0.003)$ and between those with pattern II and pattern III tumors $(P<0.05)$. However, there was no significant difference in overall survival rates between those with pattern I and pattern II hepatomas. The presence of liver cirrhosis or the presence of $\mathrm{HBsAg}$ made no statistically significant difference in predicting overall survival rates.

$S$-phase fraction. Of the 130 specimens that could be analyzed for DNA ploidy status, 104 were also evaluated for S-

Table II. Multivariate Survival Analysis in All Patients $(n=130)$

\begin{tabular}{cc}
\multicolumn{1}{c}{ Variable } & $\begin{array}{c}\text { Overall Survival } \\
P \text { value }\end{array}$ \\
\hline $\begin{array}{c}\text { Age of patient } \\
(\leq 50 \text { vs. }>50)\end{array}$ & \\
Tumor size & 0.4247 \\
$\quad(\leq 5 \mathrm{~cm}$ vs. $>5 \mathrm{~cm})$ & \\
Cut margin ${ }^{\ddagger}$ & $0.0021^{*}$ \\
$\quad(\leq 1 \mathrm{~cm}$ vs. $>1 \mathrm{~cm})$ & \\
$\quad(\leq 3 \mathrm{~cm}$ vs. $>3 \mathrm{~cm})$ & 0.1721 \\
Cirrhosis & 0.0902 \\
$\quad$ (presence vs. absence) & \\
HBsAg & 0.2015 \\
$\quad$ (positive vs. negative) & \\
Ploidy & \\
(pattern I vs. pattern II) & 0.8025 \\
(pattern I vs. pattern III) & \\
(pattern II vs. pattern III) & 0.8590 \\
\end{tabular}

+ , presence; - , absence.

* Statistically significant differences.

₹ Distance between margin of resected specimen and tumor margin.

DNA ploid distribution (pattern I, II, and III) was defined as in Methods. 
Table III. Overall Survival Analysis in Patients with Hepatomas that Could Be Analyzed with S-phase Fraction $(n=104)$

\begin{tabular}{ll}
\hline \multicolumn{1}{c}{ Variable } & $P$ value \\
\hline Pattern I vs. pattern II, low S* & 0.2104 \\
Pattern I vs. pattern II, high S & 0.1143 \\
Pattern II, low S vs. pattern III & $0.0152^{\S}$ \\
Pattern II, high S vs. pattern III & 0.6396 \\
Pattern II, low S vs. pattern II, high S & $0.0488^{\S}$ \\
Pattern I, low S vs. pattern I, high S & 0.2869 \\
\hline
\end{tabular}

${ }^{*}$ Low S, S-phase fraction $\leq 6 \%$; ${ }^{*}$ high S, S-phase fraction $>6 \%$;

$\$$ statistically significant differences.

phase fraction. Among these, 84 were pattern I and 20 were pattern II. When the prognostic importance of S-phase fraction in the 84 patients with pattern I (diploidy) hepatomas was examined, there was no statistically significant difference ( $P$ $>0.05$ ) between high and low S-phase diploid tumors in determining overall survival rate. However, by examining the effects of S-phase fraction in 20 patients with pattern II hepatoma for overall survival, there was a significant difference $(P<0.05)$ between high and low S-phase tumors, with 6\% (S-phase fraction $=6 \%$ ) being the best cutoff value. Patients with pattern II hepatomas that could be evaluated for S-phase fraction ( $n$ $=20$ ) were further compared with those who had pattern III tumors that could not be evaluated $(n=26)$. There was no notable difference in overall survival between patients who had

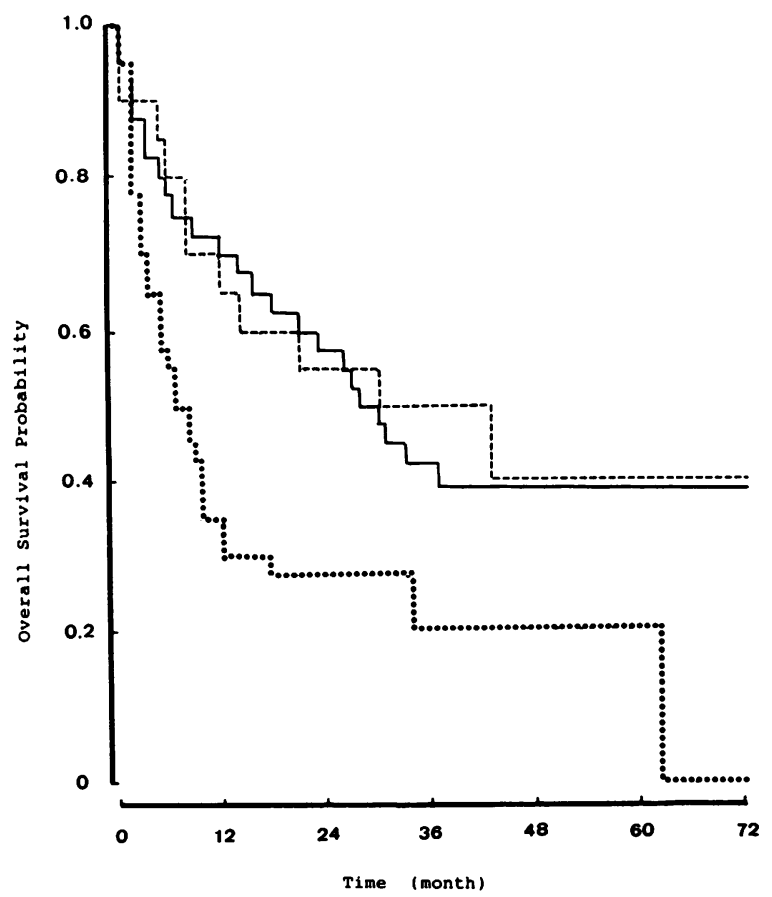

Figure 3. Overall survival probabilities according to ploidy status. Patients with pattern III hepatomas had a significantly lower rate of overall survival after hepatic resection than did those with pattern I $(P<0.003)$ or pattern II hepatomas $(P<0.05)$. Overall survival rates among the latter two groups were not significantly different. - , Pattern I $(n=84) ;--$, pattern II $(n=20) ; \cdots$, pattern III $(n=26)$. pattern II tumors with high S-phase tumors (cutoff $=6 \%$ ) and those who had pattern III hepatomas. On the contrary, patients with pattern II tumors with low S-phase tumors had higher rates of overall survival $(P<0.02)$ than did patients with pattern III hepatomas. By combining the DNA ploidy and the effects of S-phase fraction, the results of the analyses of patients with various flow-cytometric combinations are shown in Table III.

Actuarial overall survival and disease-free survival according to prognostic groups. Fig. 3 shows the actuarial overall survival for three groups of patients according to ploidy status. There were statistically significant differences in the overall survival rates between patients with pattern I and pattern III tumors $(P<0.003)$ and between those with pattern II and pattern III tumors $(P<0.05)$. The actuarial 5 -yr disease-free survival rates (Fig. 4) were all below $10 \%$.

\section{Discussion}

In this article, we have used DNA flow cytometry to analyze the DNA content of normal liver, fetal liver, focal nodular hyperplasia, and hepatomas. The results show that measurement of DNA patterns and S-phase fraction have clinical importance in predicting prognosis in patients with resectable hepatomas.

Although having a wider coefficient of variation and more cell debrils, DNA flow cytometry using paraffin blocks still has

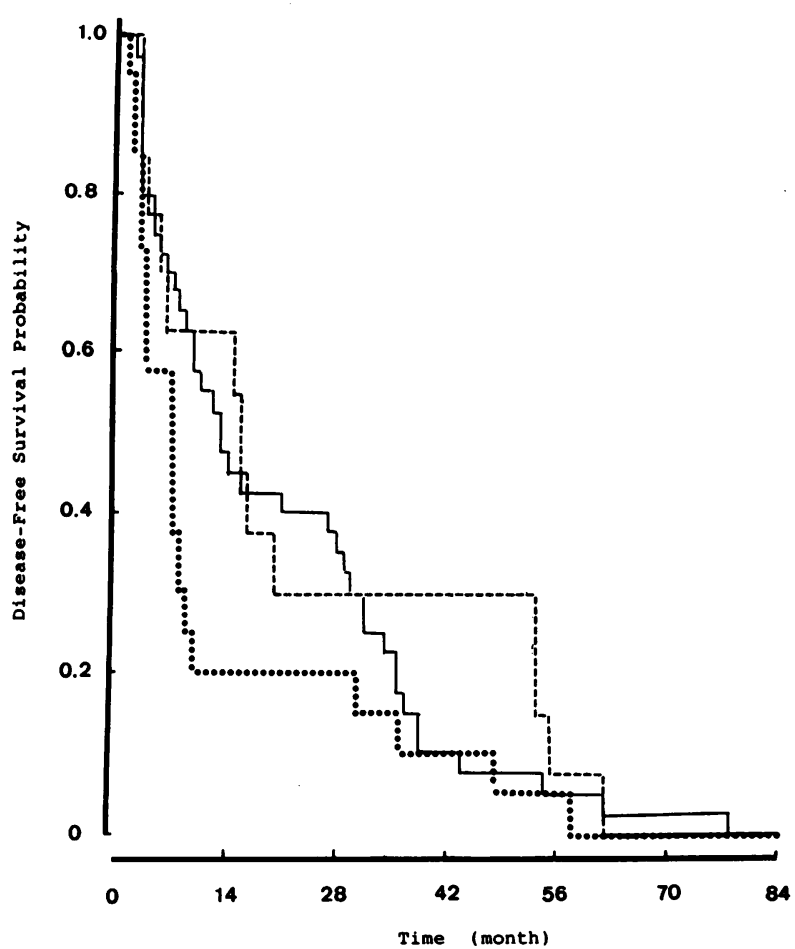

Figure 4. Disease-free survival according to ploidy status. Patients with pattern III hepatomas had a significantly lower rate of diseasefree survival after hepatic resection than did those with pattern I or pattern II tumors. Although most of the patients with hepatomas who underwent hepatic resection had tumor recurrence within five years (>90\%), two-year disease-free survival rate was $40 \%, 30 \%$, and $20 \%$ in patients with pattern I, II, and III hepatomas, respectively. Pattern I $(n=84) ;--$, pattern II $(n=20) ; \cdots$, pattern III $(n=26)$. 
good correlation between DNA indices with flow cytometry using fresh tissue (21). It is well accepted that minimized cell debrils and improved quality of DNA histograms can be obtained by using $50-\mu \mathrm{m}$ or thicker paraffin-embedded sections $(21,22)$. Besides, the abnormal DNA distributions have been more easily and more reliably identified in histograms from isolated nuclei, since these give better peak resolution than do the analyses of whole cells (15).

Previous studies have speculated on the pattern of DNA ploidy distribution of human hepatomas into two groups, diploid (euploid) and nondiploid (aneuploid), in the order of wider distribution $(14,15)$. In this study, our new system grouped hepatomas into three different patterns, pattern I, pattern II, and pattern III. Pattern I was diploid distribution (2N) as generally accepted; pattern II was aneuploid containing only one prominent G0/G1 peak, either hypoploid or hyperploid; and pattern III was aneuploid, containing more than one G0/G1 peaks. Two points about pattern III must be mentioned here. First, was there any relationship between these obvious G0/G1 peaks? Since, in addition to the obvious G0/G1 peaks, there usually existed the respective tetraploid $(4 \mathrm{~N})$ or octoploid $(8 \mathrm{~N})$ fractions, the prominent G0/G1 peaks seemed to be independent of each other. Second, was the pattern III tumor actually the pattern II tumor contaminated with normal liver parts, yielding a diploid peak in the measurement of DNA content? It is worthy of mention that the specimens were managed at the time when the tumors were resected and carefully treated to avoid obtaining specimens with necrosis or containing nontumor parts. Besides, a prominent diploid $\mathrm{G} 0 / \mathrm{G} 1$ peak with an aneuploid G0/G1 peak, and, moreover, two aneuploid peaks, could sometimes be obtained in pattern III tumors. Therefore, contamination of normal hepatocytes to yield a pattern III ploidy distribution, i.e., pattern II (tumor DNA ploidy), mixed with normal hepatocytes (diploid) is not likely.

The DNA pattern, separated into diploid and nondiploid groups, has been reported to correlate with tumor size and age of patients but not with the presence of $\mathrm{HBsAg}$ or the presence of liver cirrhosis. Furthermore, it was also suggested that there was no significant correlation between DNA pattern and the overall survival rates of hepatoma patients who underwent hepatic resection (14). In our study, there was no correlation among DNA pattern and tumor size, age of patients, presence of $\mathrm{HBsAg}$, or presence of liver cirrhosis. Similarly, if the DNA pattern was separated into diploid and nondiploid groups, there was no correlation between DNA pattern and the prognosis of patients who underwent liver resection. However, when the DNA ploidy status is stratified into three patterns, poor prognosis could be observed in patients with pattern III ploidy in terms of overall survival and disease-free survival (Figs. 3 and 4). Although most of the patients with hepatomas after resection had tumor recurrence within five years $(>90 \%)$, the two-year disease-free survival was $40 \%, 30 \%$, and $20 \%$ in patients with pattern I, II, and III hepatomas, respectively. Also, considering the possibility of selection bias, patients receiving palliative resection (no disease-free survival) were followed up and the mean overall survival times were $12.67 \pm 3.74$ months and 5.14 \pm 1.32 months in those with pattern I and pattern III tumors, respectively (no patients with pattern II hepatomas received palliative resection). The difference between these two groups seemed to be statistically insignificant $(P=0.08)$. We attributed this to inadequate sample size, and further work on the palliative resection group is ongoing. This result suggests that hepatomas with pattern III ploidy status have more malignant biological behavior than those with pattern I and II ploidy status.

It has been proposed that aneuploid cells may represent distinct tumor cell populations that carry their own stem lines and that the increased tendency toward diploid divisional proliferation may favor the subsequent development of aneuploid subpopulations, a process similar to that described for epithelial or lymphoid tissues $(23,24)$. In pattern III tumors, two possibilities can be suggested. First, the tumor cells are of single clonal origin with development of aneuploid subpopulations. Second, the tumor cells originate from more than one stem line. The exact nature of these tumors needs further work at the molecular level.

Although S-phase fraction played a role in the overall survival of patients with pattern II ploidy hepatoma, it had no effect on those with pattern I or diploid tumors. In addition, the lack of statistical significance in overall survival rate between patients with pattern I (diploidy) and pattern II hepatomas suggested that the prognosis of patients with hepatoma seemed to be influenced by other factors, such as tumor size, surgical technique, liver cirrhosis, etc., as previously reported (14), and by tumor size in the present work. Nevertheless, measurement of DNA ploidy and estimation of S-phase fraction in hepatoma patients may be helpful in determining treatment strategies, as demonstrated in treatment of breast cancer (25).

\section{Acknowledgments}

We are indebted to Dr. Chungming Chang for his advice and helpful discussion in preparation of this manuscript.

\section{References}

1. Health statistics. 1979. V. II. National Health Administration, Taiwan Provincial Health Department and Taipei City Health Department, Republic of China. 34-137.

2. Hunt, D. D. 1963. Primary liver cell carcinoma with a protracted clinical course. JAMA (J. Am. Med. Assoc.). 184:146-148.

3. Yoshida, T., N. Okazaki, M. Yoshino, H. Kitaoka, S. Hirohashi, and Y. Shimosato. 1982. Minute hepatocellular carcinoma without appreciable change in size for seven years: a case report. Cancer (Phila.). 49:1491-1495.

4. Watson, J. V. 1986. Oncogenes, cancer and analytical cytology. Cytometry. 7:400-410.

5. Wied, G. L., P. H. Bartels, H. E. Dytch, and M. Bibbo. 1983. Rapid DNA evaluation in clinical diagnosis. Acta Cytol. 27:33-37.

6. Watson, J. V. 1988. Oncogene expression in diagnosis and prognosis in human malignancy (meeting abstract). Cytometry. 2(Suppl.):6.

7. Young, G. A. R., D. W. Hedley, C. A. Rugg, and H. J. Iland. 1987. The prognostic significance of proliferative activity in poor histology non-Hodgkin's lymphoma: a flow cytometry study using archival material. Eur. J. Cancer \& Clin. Oncol. 23:1497-1504.

8. Ewers, S. B., E. Langstrom, B. Baldetorp, and D. Killander. 1984. Flow-cytometric DNA analysis in primary breast carcinomas and clinicopathological correlations. Cytometry. 5:408-419.

9. Schwarze, P. E., E. O. Pettersen, M. C. Shoaib, and P. O. Seglen. 1984. Emergence of a population of small, diploid hepatocytes during hepatocarcinogenesis. Carcinogenesis (Eynsham). 5:1267-1275.

10. Deleener, A., P. Castelain, V. Preat, J. Gerlache, H. Alexandre, and M. Kirsch-Volders. 1987. Changes in nucleolar transcriptional activity and nuclear DNA content during the first steps of rat hepatocarcinogenesis. Carcinogenesis (Eynsham). 8:195-201.

11. Saeter, G., P. E. Schwarze, J. M. Nesland, N. Juul, E. O. Pettersen, and P. O. Seglen. 1988. The polyploidizing growth pattern of normal rat liver is replaced by divisional, diploid growth in hepatocellular nodules and carcinomas. Carcinogenesis (Eynsham). 9:939-945. 
12. Mori, H., T. Tanaka, S. Sugie, M. Takahashi, and G. M. Williams. 1982. DNA content of liver cell nuclei of N-2-fluorenylacetamide-induced altered foci and neoplasms in rats and human hyperplastic foci. J. Natl. Cancer Inst. (Bethesda). 69:1277-1282.

13. Sarafoff, M., H. M. Rabes, and P. Dormer. 1986. Correlations between ploidy and initiation probability determined by DNA cytophotometry in individual altered hepatic foci. Carcinogenesis (Eynsham). 7:1191-1196.

14. Ezaki, T., T. Kanematsu, T. Okamura, T. Sonoda, and K. Sugimachi. 1988. DNA analysis of hepatocellular carcinoma and clinicopathologic implications. Cancer (Phila.). 61:106-109.

15. Saeter, G., C. Z. Lee, P. E. Schwarze, S. Ous, D. S. Chen, J. L. Sung, and P. O. Seglen. 1988. Changes in ploidy distributions in human liver carcinogenesis. J. Natl. Cancer Inst. (Bethesda). 80:1480-1485.

16. Clark, G. M., L. G. Dressler, M. A. Owens, G. Pounds, T. Oldaker, and W. L. McGuire. 1989. Prediction of relapse or survival in patients with nodenegative breast cancer by DNA flow cytometry. $N$. Engl. J. Med. 320:627-633.

17. Dressler, L. G., L. Seamer, M. A. Owens, G. M. Clark, and W. L. McGuire. 1987. Evaluation of a modeling system for S-phase estimation in breast cancer by flow cytometry. Cancer Res. 47:5294-5302.

18. Coon, J. S., A. L. Landay, and R. S. Weinstein. 1986. Flow cytometric analysis of paraffin embedded tumors. Implications for diagnostic patbology. Hum. Pathol. 117:435-437.

19. Kaplan, E. L., and P. Meier. 1958. Nonparametric estimation from incomplete observations. J. Am. Stat. Assoc. 53:457-481.

20. Cox, D. R. 1972. Regression models and life-tables. J. R. Stat. Soc. (B). 34:187-220.

21. Hedley, D. W. 1989. Flow cytometry using paraffin-embedded tissue: five years on. Cytometry. 10:229-241.

22. Stephenson, R. A., H. Gay, W. R. Fair, and M. R. Melamed. 1986. Effect of section thickness on quality of flow cytometric DNA content determinations in paraffin-embedded tissue for flow cytometry. Cytometry. 7:41-44.

23. Mauro, F., L. Teodori, J. Schumann, and W. Gohde. 1986. Flow cytometry as a tool for the prognostic assessment of human neoplasia. Int. J. Radiat. Oncol. Biol. Phys. 12:625-636.

24. Friedlander, M. L., D. W. Hedley, and I. W. Taylor. 1984. Clinical and biological significance of aneuploidy in human tumours. J. Clin. Pathol. (Lond.). 7:961-974.

25. Bonadomma, G., P. Valagussa, and G. Tancini. 1986. Current status of Milan adjuvant chemotherapy trials for node-negative breast cancer. Natl. Cancer Inst. Monogr. 1:45-49. 